\title{
PARTICIPAÇÃO DO PACIENTE COM DORES CRÔNICAS NA CONSTRUÇÃO DA CONDUTA MÉDICA E ADESÃO AO TRATAMENTO
}

Rafael de Oliveira Pena Neto, José de Oliveira Costa Filho, Marjori Leiva Camparoto, Tamara Batata Silva, Rafaela Gimenes Mussi, Letícia Moraes Lira, Marina de Souza Bastos Lino, André Mio Takayama

Universidade do Oeste Paulista - Faculdade de Medicina, Presidente Prudente, SP. e-mail: rafaelpneto@hotmail.com. Agência de fomento: Conselho Regional de Medicina do Estado de São Paulo.

\section{RESUMO}

No tratamento de dores crônicas é difícil a definição de um modelo específico para abordagem terapêutica, sendo importante a avaliação individual de cada paciente com participação ativa do mesmo. No Brasil, ainda são escassos os estudos sobre a participação do indivíduo na construção da conduta. O presente estudo teve como objetivo avaliar a associação entre a adesão à terapêutica prescrita e o grau de participação dos doentes na construção da conduta médica. Cerca de $87,5 \%$ dos pacientes que abandonaram um tratamento não haviam participado da construção do mesmo, o que denota uma correlação positiva entre os a autonomia e a adesão ao tratamento $(p=0,004)$. Apesar da maioria dos pacientes ter conhecimento sobre detalhes da sua doença, ainda está instalado um modelo não participativo, que institui somente ao médico a tomada de decisão, já que estes não são questionados pelo médico quanto as suas necessidades e dificuldades.

Palavras-chave: autonomia pessoal, bioética, automedicação, dor crônica, saúde holística.

\section{PARTICIPATION OF THE PATIENT WITH CHRONIC PAIN IN THE CONSTRUCTION OF MEDICAL DECISION AND TREATMENT ACCEPTANCE}

\begin{abstract}
In the treatment of chronic pain it is difficult to define a specific model for therapeutic approach, being important the individual evaluation of each patient with active participation of the same. In Brazil, studies on the participation of the patient in the construction of the treatment are still scarce. The present study aimed to evaluate the association between adherence to therapy and the degree of participation of patients in the construction of medical conduct. Approximately $87.5 \%$ of the patients who abandoned treatment did not participate in the construction, which indicates a positive correlation between autonomy and adherence to treatment $(p=0.004)$. Although most patients are aware of the details of their disease, a non-participatory model is established, which only establishes the decision-making process for the physician, since these are not questioned by the physician about their needs and difficulties.
\end{abstract}

Keywords: personal autonomy, self-medication, bioethics, chronic pain, holistic health.

\section{INTRODUÇÃO}

A RESOLUÇÃO CFM N 1.931, de 17 de setembro de 2009 institui o Código de Ética Médica, contendo as normas que devem ser seguidas pelos médicos no exercício de sua profissão. O Capítulo I descreve os princípios fundamentais da prática médica, estando no Artigo XXI a seguinte afirmação: "No processo de tomada de decisões profissionais, de acordo com seus ditames de consciência e as previsões legais, o médico aceitará as escolhas de seus pacientes, relativas aos procedimentos diagnósticos e terapêuticos por eles expressos, desde que adequadas ao caso e cientificamente reconhecidas" ${ }^{1}$.

Em busca de um tratamento mais adequado, que enquadre as necessidades e anseios dos pacientes, surge o termo autonomia. Esse termo significa a capacidade de se autogovernar, ou seja, o indivíduo pode realizar 
suas escolhas de forma autônoma, desde que sejam capazes de agir intencionalmente. Para que uma decisão seja feita de forma autônoma, além da capacidade, o indivíduo deve ter liberdade e conhecimento sobre os objetivos e consequências das ações que serão tomadas ${ }^{2}$. Paulo Freire ${ }^{3}$ propõe a seguinte afirmação: "O respeito à autonomia e à dignidade de cada um é um imperativo ético e não um favor que podemos ou não conceder uns aos outros".

A insatisfação do paciente perante um atendimento superficial, impessoal e que não o esclareça sobre sua condição, é um fator importante para abandono do tratamento e a prática da automedicação ${ }^{4}$. Ao considerar que o paciente possui uma participação determinante no processo de um tratamento, a adesão e não adesão à terapêutica constitui-se um fenômeno multifatorial e complementar, que uma vez interrelacionados, podem produzir melhores ou piores resultados no tratamento ${ }^{5}$.

Ao possibilitar que o paciente aja com autonomia perante seu tratamento, o profissional, além de estar cumprindo um dever ético, também promove maiores chances de uma boa aceitação à terapêutica prescrita ${ }^{6}$. Devido à necessidade do uso de condutas prolongadas para o tratamento de dores crônicas, é de extrema importância para os profissionais da saúde, deixar de ver o paciente como mero objeto da intervenção terapêutica tecnicista. Mais do que a postura passiva do paciente frente ao tratamento estipulado, o sucesso e aceitação da terapia implica postura ativa do indivíduo frente ao autocuidado ${ }^{7}$.

A literatura tem apontado que a autonomia tem grande influência na adesão do tratamento pelo paciente. Diante disto, o presente estudo tem como objetivo geral avaliar a associação entre a adesão à terapêutica prescrita e o grau de participação dos doentes na construção da conduta médica em pacientes com queixa de dores crônicas.

\section{METODOLOGIA}

O presente estudo tem caráter prospectivo do tipo coorte transversal e foi desenvolvido em pacientes que buscaram atendimento ambulatorial num o hospital público localizado em Presidente Prudente - SP, com queixa de dor crônica. $O$ presente projeto foi aprovado pelo Comitê de Ética em Pesquisa da
Universidade do Oeste Paulista - UNOESTE (CAAE: 55863116.0.0000.5515). Para o estudo foram incluídos todos os pacientes com idade acima de 18 anos que receberam atendimento ambulatorial no hospital e que assinaram o Termo de Consentimento Livre e Esclarecido ( $T C L E$ ) em anexo e que tiveram queixas de dores crônicas, sem distinção de fatores causais. A técnica utilizada para a coleta de dados foi a entrevista individual entre um aplicador e um participante. $O$ instrumento utilizado foi roteiro de entrevista com questões abertas e fechadas. A análise dos dados foi de cunho quali-quantitativo. Para o estudo das associações foi utilizado o teste de Qui-quadrado de Pearson ou Exato de Fisher. As razões de chances (Odds-ratio) por ponto e por intervalos com $95 \%$ de confiança e nível de significância de $5 \%$.

\section{RESULTADOS}

No total, foram entrevistados 74 pacientes, estando todos enquadrados nos critérios de inclusão estabelecidos. Destes, 54 são do sexo feminino $(72,97 \%)$ e 20 do sexo masculino $(27,03 \%)$, com média etária de $53,7 \pm$ 13,3 anos, variando entre 18 e 85 anos. Segundo a raça, $45,9 \%$ consideravam-se brancos, $36,48 \%$ pardos, $16,22 \%$ negros e $1,35 \%$ amarelos. Considerando a religião declarada pelos entrevistados, 41 eram católicos, 23 evangélicos, 3 ateus e 7 seguem outras religiões. As doenças ortopédicas e reumatológicas foram as patologias em tratamento mais prevalentes dentre os entrevistados.

A Tabela 1 descreve a frequência de respostas dos entrevistados para questionamentos que indicam a postura do médico frente ao manejo do tratamento do paciente com dor crônica.

Com relação à manutenção adequada do tratamento, a confiança no médico foi indicada por 56 entrevistados $(75,68 \%)$, o custo da medicação por 10 (13,51\%), participação na escolha do tratamento por $6(8,11 \%)$ e tempo de tratamento por $2(2,70 \%)$. Diante das perguntas que dizem respeito a autonomia do paciente frente ao tratamento prescrito, $89,19 \%(n=66)$ consideram importante a sua participação na escolha do seu tratamento. Número esse, assemelha-se aos que gostariam de participar de forma autônoma e conjunta na escolha da terapia prescrita $(89,19 \%)$. Apenas, $10,81 \%(n=8)$, 
não gostariam de participar e nem consideram importante sua contribuição na construção da conduta médica. Por outro lado, $87,1 \%$ dos pacientes que demonstraram desejo em participar, responderam que o médico pergunta sua opinião sobre o tratamento "às vezes", "raramente" ou "nunca".

Tabela 1. Frequência de resposta às perguntas relacionadas à postura do médico frente ao manejo do tratamento $(n=74)$.

\begin{tabular}{|c|c|c|c|c|c|}
\hline & Nunca & Raramente & Às vezes & Geralmente & Sempre \\
\hline $\begin{array}{l}\text { 1. O médico explica a você detalhes } \\
\text { sobre a sua doença? }\end{array}$ & $\begin{array}{l}21,62 \% \\
(n=16)\end{array}$ & $\begin{array}{c}10,81 \% \\
(n=8)\end{array}$ & $\begin{array}{l}16,22 \% \\
(n=12)\end{array}$ & $\begin{array}{l}24,32 \% \\
(n=18)\end{array}$ & $\begin{array}{l}27,03 \% \\
(n=20)\end{array}$ \\
\hline 2. O médico propõe diferentes tipos & & & & & \\
\hline $\begin{array}{l}\text { de tratamento para que você participe } \\
\text { da escolha? }\end{array}$ & $\begin{array}{c}48,65 \% \\
(n=36)\end{array}$ & $\begin{array}{l}21,62 \% \\
(n=16)\end{array}$ & $\begin{array}{c}2,70 \% \\
(n=2)\end{array}$ & $\begin{array}{c}10,81 \% \\
(n=8)\end{array}$ & $\begin{array}{l}16,22 \% \\
(n=12)\end{array}$ \\
\hline 3. O médico pergunta sua opinião & & & & & \\
\hline $\begin{array}{l}\text { sobre o tratamento que ele está } \\
\text { prescrevendo? }\end{array}$ & $\begin{array}{l}62,16 \% \\
(n=46)\end{array}$ & $\begin{array}{c}10,81 \% \\
(n=8)\end{array}$ & $\begin{array}{c}10,81 \% \\
(n=8)\end{array}$ & 0 & $\begin{array}{l}16,22 \% \\
(n=12)\end{array}$ \\
\hline 4. O médico pergunta sobre as & & & & & \\
\hline encontradas & $56,76 \%$ & $5,41 \%$ & $2,70 \%$ & $10,81 \%$ & $24,32 \%$ \\
\hline $\begin{array}{l}\text { tratamento prescrito na última } \\
\text { consulta? }\end{array}$ & $(n=42)$ & $(n=4)$ & $(n=2)$ & $(n=8)$ & $(n=18)$ \\
\hline
\end{tabular}

Dos entrevistados, 16 relataram já ter abandonado algum tratamento médico, enquanto 58 negam essa prática. Cerca de $87,5 \%$ dos pacientes que abandonaram um tratamento não haviam participado da construção do mesmo, o que denota uma correlação positiva entre os a autonomia e a adesão ao tratamento $(p=0,004)$. Semelhantemente, comprovou-se significância entre a maior taxa de abandono em pacientes em que o médico não pergunta sua opinião sobre o tratamento $(p<0,001)$.

As justificativas dadas pelos entrevistados a dois questionamentos serão distribuídas em três categorias para melhor compreensão da real ideia dos mesmos frente à importância e desejo de participarem ativamente na construção da conduta médica.

"Você acha importante sua participação na escolha do seu tratamento?".

1- Necessidade em expressar suas experiências prévias: diante dos relatos dados durante as entrevistas, pôde-se perceber 0 anseio dos pacientes em contar ao médico suas experiências prévias com tratamentos já realizados. Nota-se que uma postura mais passiva do médico, permitindo que o paciente fale, é um fator contribuinte para o crescimento da confiança no médico.
2- Preocupação quanto aos efeitos do tratamento: por serem os indivíduos que receberão 0 tratamento, muitos pacientes mostram-se interessados em participar da construção da conduta médica. Conhecer mais sobre sua doença e sobre os efeitos dos medicamentos prescritos também são itens relevantes para os pacientes.

3- Visão voltada para o conhecimento médico: apesar de representar a minoria dos pacientes, alguns ainda desvalorizam sua participação. Por acreditar que o conhecimento técnico do médico, a respeito da sua doença, é maior, eles possuem nada a acrescentar na escolha do tratamento. Eles depositam no médico toda confiança e responsabilidade frente ao que será prescrito.

"Você gostaria de participar da escolha do seu tratamento?".

1- Anseio em expressar as dificuldades encontradas: cerca de $66 \%$ dos pacientes relatam que o médico nunca pergunta a eles sobre as dificuldades encontradas durante 0 tratamento. Estes dados corroboram com a fala de muitos pacientes, visto que eles exprimem o desejo de contar ao médico quais as dificuldades encontradas no período 
interconsulta, seja ela dúvidas sobre os efeitos dos medicamentos, efeitos colaterais ou dificuldade em adquirir o medicamento.

2- Autonomia vista como um direito do paciente: alguns entrevistados mostraram em suas falas, percepções em relação a sua autonomia, visto que colocam sua participação como algo de direito. Acreditando-se que muitos não conhecem o Código de Ética Médica, nota-se que os pacientes querem participar ativamente e ser respeitados conforme suas limitações.

3- Desvalorização das experiências vividas: os mesmos pacientes que expressaram a não importância da sua participação autônoma na construção tratamento, também não expressaram o desejo de participar.

\section{DISCUSSÃO}

A amostra da pesquisa mostrou-se bastante heterogênea, sendo possível assim, analisar a fala de pacientes de diferentes sexos, etnias, faixas etárias e religiões. É de extrema importância notar as diferentes opiniões e angústias que cada indivíduo tem diante do seu tratamento.

Cerca de $84 \%$ da amostra queixa-se de dores crônicas há mais de 18 meses. As patologias ortopédicas, como hérnia de disco, lombalgia e osteoartrose são as mais prevalentes na população estudada, seguido de patologias de origem reumatológica como artrite reumatoide e fibromialgia e de origem neurológica, como enxaqueca. Frequentemente, estas patologias demandam de um tratamento contínuo e prolongado, que por vezes são ad eternum. Desta forma, estão mais predispostos ao desenvolvimento de psicopatologias como ansiedade e depressão, que colaboram negativamente no autocuidado ${ }^{8,9}$.

Aproximadamente, $50 \%$ dos pacientes procuram, inicialmente, atendimento no posto de saúde ao sentirem dores, mostrando a importância de serviços de saúde instalados dentro das comunidades. Entretanto, ainda existe pacientes que procuram atendimento em farmácias e outros serviços não médicos para a prescrição de medicamentos analgésicos, o que pode ser prejudicial à saúde do mesmo ${ }^{10}$.

Apesar de apenas $75,67 \%$ dos pacientes relatarem buscar, como primeira escolha, o atendimento em postos de saúde, hospitais ou consultório médico, cerca de $70 \%$ dos entrevistados relataram já terem praticado a automedicação ao sentirem dores. Ao serem interrogados sobre o motivo que levaram a essa prática, a grande maioria relatou o uso de analgésicos por conta própria, pois a dor era muito intensa e queriam alívio rapidamente.

Fatores ligados à experiência pregressa do pacientes com o medicamento e ao baixo conhecimento em saúde estão fortemente ligados à automedicação ${ }^{11}$. Além disso, notou-se maiores índices de automedicação em pacientes de médicos que não se interessavam sobre as dificuldades encontradas com o tratamento no período interconsulta.

Ao possibilitar a participação do paciente na construção da conduta médica, obtêm-se melhores resultados na qualidade de vida do paciente e na aceitação da terapia prescrita ${ }^{6}$. Comumente, pacientes com dores crônicas necessitam de tratamento contínuo e com inúmeros medicamentos. Sabendo-se que as experiências com fármacos utilizados anteriormente pelo paciente é um fator ligado ao uso adequado ou não dos mesmos ${ }^{12}$, têm-se resultados contraditórios na amostra pesquisada. A maior parte dos participantes relatou que o médico não pergunta sua opinião sobre o tratamento, tão quanto, propõe diferentes opções terapêuticas. $O$ conhecimento do paciente acerca da sua doença é um fator que prediz maior qualidade no autocuidado e no tratamento ${ }^{12}$. Nesse estudo, 51,35\% responderam que o médico explica a eles detalhes sobre suas patologias, possibilitando que o doente tenha capacidade crítica em compreender as alterações em seu corpo.

$\mathrm{Na}$ análise dos fatores que contribuem para o abandono do tratamento, foi constatado que há maiores índices de desistência em pacientes que não participaram da construção do seu tratamento. A correlação entre maiores índice de abandono do tratamento em pacientes que o médico não pergunta sua dificuldades é real, demonstrando a influência positiva no seguimento adequado do tratamento, em doentes que tem sua autonomia respeitada ${ }^{5}$. 
Em toda a amostra, o principal fator contribuinte para manutenção adequada do tratamento foi a confiança no médico. Segundo Ugarti e Acioly ${ }^{13}$, a confiança no médico e a oportunidade de participar das decisões aumentam os índices de satisfação do paciente com o tratamento. Entre os pacientes que abandonaram o tratamento, o principal fator, também, foi a confiança no médico.

A análise referente à opinião do paciente frente à importância da sua participação e do seu desejo em participar mostrou que a grande maioria $(89,9 \%)$ reconhece seu direito à autonomia na escolha do tratamento e gostaria de colocá-la em prática. Entretanto, uma parcela menor dos pacientes $(10,1 \%)$, tiveram opiniões contrárias, ou seja, não gostariam de participar. Isso acontece, pois os mesmos não consideram sua opinião significativa e não reconhecem sua autonomia como um fator contribuinte para o sucesso da sua terapia.

\section{CONCLUSÃO}

O seguimento adequado do tratamento é diretamente ligado à participação autônoma do paciente na construção do mesmo. Em pacientes que não participaram ativamente da construção do seu tratamento, observou-se maiores taxas de abandono. E se tratando de pacientes com dores crônicas, quando é realizada uma consulta não participativa, há maiores índices de automedicação, o que pode trazer riscos à saúde do indivíduo.

Apesar da maioria dos pacientes terem conhecimento sobre detalhes da sua doença, ainda está instalado um modelo não participativo, que institui somente ao médico a tomada de decisão, já que estes não são questionados pelo médico quanto as suas necessidades e dificuldades.

Embora alguns pacientes não considerem importante um modelo participativo, a imensa maioria considera importante e gostaria de participar da construção da conduta médica. Entretanto, a sua participação não é vista como principal fator contribuinte para manutenção do tratamento, e sim, a confiança no médico.

\section{CONFLITO DE INTERESSES}

Os autores declararam não haver qualquer potencial conflito de interesse que possa interferir na imparcialidade deste trabalho cientifico.

\section{REFERÊNCIAS}

1. Conselho Federal de Medicina. Código de Ética Médica. São Paulo; 2010. 31p.

2. Beauchamp TL, Childress JF. Principles of biomedical ethics. 7.ed. New York: Oxford; 2013.

3. Freire P. Pedagogia da autonomia: saberes necessários à prática educativa. 43.ed. São Paulo: Paz e Terra; 2011.

4. Naves JOS, Castro LLC, Carvalho CMS, Hamann EM. Automedicação: uma abordagem qualitativa de suas motivações. Ciênc Saúde Coletiva 2010;15(Supl 1):1751-62. DOI: https://doi.org/10.1590/S1413$\underline{81232010000700087}$

5. Cunha MF, Gandini RC. Adesão e não-adesão ao tratamento farmacológico para depressão. Psicol Teoria Pesq. 2009;25(3):409-18. DOI: https://doi.org/10.1590/S0102-37722009000300015

6. Bitterncourt ALP, Quintana AM, Velho MTAC, Goldim JR, Wottrich LAF, Cherer EQ. A voz do paciente: por que ele se sente coagido? Psicol Estud. 2013;18(1):93-101.

DOI:

https://doi.org/10.1590/S1413-73722013000100010

7. Gusmão JL, Mion D. Adesão ao tratamento: conceitos. Rev Bras Hipert. 2006;13(1):23-5.

8. Canadian Agency for Drugs and Tecnology in Health. Diagnosing, screening, and monitoring depression in the elderly: a review of guidelines. Rapid Response Record: Summary with Critical Appraisal 2015;1:1-20.

9. Dennis M, Kadri A, Coffey J. Depression in older people in the general hospital: a systematic review of screening instruments. Age Ageing. 2012;41(2):14854. DOI: https://doi.org/10.1093/ageing/afr169

10. Tierling VL, Paulino MA, Fernandes LC, Schenkel EP, Mengue SS. Level of knowledge of the compositions of analgesic medication containing aspirin. Rev Saúde Pública. 2004;38(2):223-7. DOI: https://doi.org/10.1590/S0034-89102004000200011

11. Linsky A, Simon SR, Stolzamann K, Bokhour BG, Meterko M. Prescribers' perceptions of medication discontinuation: survey instrument development and validation. Am J Manag Care. 2016;22(11):747-54.

12. Veenker $\mathrm{H}$, Paans W. A dynamic approach to communication in health literacy education. BMC Med Educ. 2016;16(1):1-12. DOI: https://doi.org/10.1186/s12909-016-0785-z 
13. Ugarte ON, Acioly MA. The principle of autonomy in Brazil: one needs to discuss it. Rev Col Bras Cirurg. 2014;41(5):374-7. DOI: https://doi.org/10.1590/0100$\underline{69912014005013}$

Recebido para publicação em 08/08/2017

Revisado em 29/08/2017

Aceito em 26/09/2017 